\title{
Engaging for Puerto Rico: \#RickyRenuncia (and \#RickySeQueda) during El Verano del 19 and digital identities
}

\author{
Perez-Rivera, Danilo T. (University of Puerto Rico) \\ Torres-Lugo, Christopher (Indiana University, Bloomington) \\ Santos-Lozada, Alexis R. (Pennsylvania State University)
}

\section{Corresponding Author:}

Danilo T. Perez-Rivera

University of Puerto Rico, Medical Sciences Campus

Email: danilo.perez@upr.edu

\begin{abstract}
Between July 13-24, 2019 the people of Puerto Rico took the streets after a series of corruption scandals shocked the political establishment. The social uprising resulted in the ousting of the Governor of Puerto Rico (Dr. Ricardo Rosselló, Ricky), the resignation of the majority of his staff something unprecedented in the history of Puerto Rico; this period has been called El Verano del 19 (Summer of 19). Social media played a crucial role in both the organization and dissemination of the protests, marches, and other activities that occurred within this period. Puerto Ricans in the island and around the world engaged in this social movement through the digital revolution mainly under the hashtag \#RickyRenuncia (Ricky Resign), with a small counter movement under the hashtag \#RickySeQueda (Ricky will stay). The purpose of this study is to illustrate the magnitude and grass roots nature of the political movement's social media presence, as well as their characteristics of the population of both movements and their structures. We found that \#RickyRenuncia was used approximately one million times in the period of analysis while \#RickySeQueda barely reached 6,000 tweets. Particularly, the pervasiveness of cliques in the \#RickySeQueda show concentrations of authority dedicated to its propagation, whilst the \#RickyRenuncia propagation was much more distributed and decentralized with little to no interaction between significant nodes of authority. Noteworthy was the role of the Puerto Rican diaspora in the United States of America and around the world, contributing close to $40 \%$ of all geo-located tweets. Finally, we found that the Twitter followers of the former governor had indicators of being composed of two distinct populations: 1) those active in social media and 2) those who follow the account but who are not active participants of the social network. We discuss the implications of these findings on the interpretation of emergence, structure and dissemination of social activism and countermovement to these activities in the context of Puerto Rico.
\end{abstract}

DO NOT CITE WITHOUT AUTHORIZATION OF THE AUTHORS 


\section{Introduction}

On July 13, 2019, the Centro de Periodismo Investigativo (CPI, Center for Journalistic Research) published 889 pages of a Telegram chat between the Governor of Puerto Rico (Dr. Ricardo Rosselló, also known as Ricky) and his closest circle. The pages were filled with derogatory, misogynistic, homophobic, and discriminatory language geared towards anybody who was deemed a critic of the government (Valentín Ortiz \& Minet, 2019). The members of this chat included public relations specialists, the Chief of Staff for Puerto Rico, the Secretary of the Treasury, the Secretary of Public Affairs and a lobbyist; the latter had privileged information to public policy discussions through this chat. For the people of Puerto Rico this spark ignited " $E l$ Verano del 19" (The Summer of 19) with calls and manifestations in the island and around the world, which caused the resignation of the Governor of Puerto Rico and, most of the participants of the chat (CPI). El Verano del 19 also sparked social media engagement supporting, and to a lesser extent against, the ousting of the Governor.

Similar to the case of Spain's 15M, Twitter, and social media in general, became the tool for organization of protests and marches (Anduiza, Cristancho, \& Sabucedo, 2014). Other example includes the role that social media and the Arab Spring, which has been studied previously (Wolfsfeld, Segev, \& Sheafer, 2013); research that underscores the importance of social networks and social media in recent social movements. In Puerto Rico, the coordination of protests and creative manifestations mostly relied on a user posting an image to social media calling for a specific type of protest, a date and time, and a meeting place. The types of the protests were wideranging, from yoga, to horseback riding, to even a perreo combativo (combative reggaeton dancing). Figure 1 includes a compilation of four images that were used to summon persons to protest: 1) Grito de Fortaleza, 2) New York Show up Announcement, 3) Yoga en Fortaleza, and 4) Perreo Combativo. The Governor's resignation was announced during the event known as perreo combativo. All of these manifestations were a success in terms of attendance, even though nobody coordinated them activities in an official capacity.

In terms of social media manifestations, protestors relied on these platforms to let the world know of their demands for the governor to resign. Tuitazos, tweet storms, where users would all publish tweets using the same hashtag at a designated time, were frequently organized around a specific hashtag. Each of these tuitazos were based around a different hashtag in an attempt to defeat Twitter's trending topic model, which places limits on how often a hashtag can become a trend. Many, if not all, of these hashtags became trends, some of them even at a national or worldwide scale. Social media also served to document second-by-second what was happening at not only La Fortaleza, the governor's mansion that was the epicenter of the protests, but also around the island and world where Puerto Ricans also carried out protests. This endless stream of documentation served to combat government misinformation that sought to blame protestors for the violence carried out by the police whenever they decided that it was time to disperse the protests. Tweet storms are discussed in previous literature that studies the influence of social media in political participation (Varnali \& Gorgulu, 2015). 


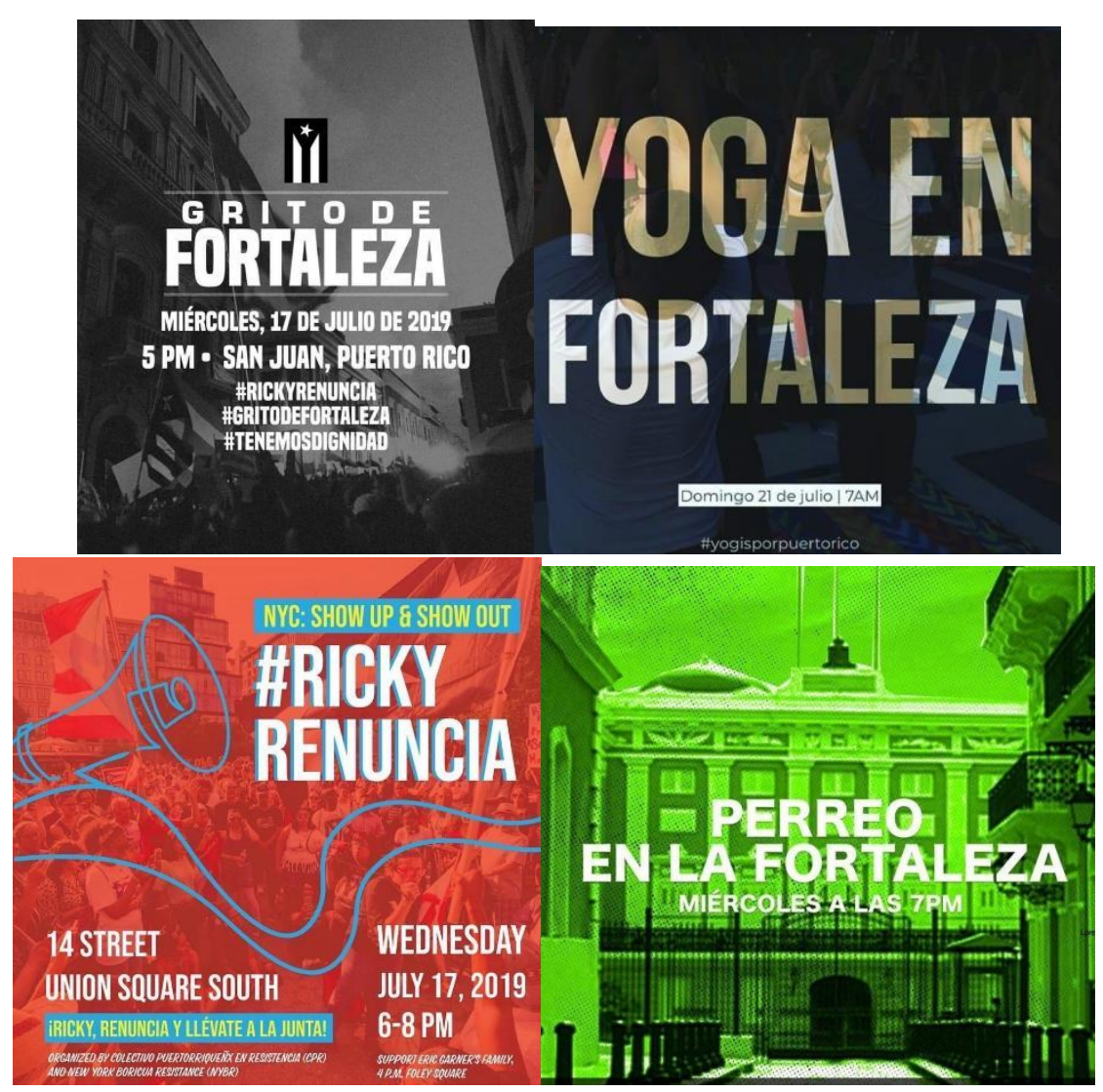

Figure 1: Pictures shared through social media to call for specific manifestations.

Similar to Donald Trump, Twitter was a crucial tool for dissemination used by the former Governor. It was common to see pictures of official meetings ("Aqui Reunidos" or "Reunidos"), official communications were shared on this accounts, and the Governor had ";Buenos Dias!" messages scheduled every morning some of which were answered in less than one minute by those who awaited these messages (See Figure 2). Twitter was also used as a battleground by professional spinners and a group of accounts would harass those who were not in line with the official narrative or who contradicted the official information (Valentín Ortiz \& Minet, 2019). A recent forensic analysis indicated that indeed some accounts on Twitter were acting with a mathematical concordance with the wishes of the governor as expressed in the leaked chats (Bandeira, Ponce de León, \& Clark, 2019). The behaviors, described as "bot like" had been a topic of discussion among many members of the community known as \#TwitterPR for the last two years. 
Ricardo Rossello

@ricardorossello

¡Buenos días!

$11 / 24 / 18,7: 30 \mathrm{AM}$

3 Retweets 17 Likes

坆 0 个

Panel A: Tweet after 3 minutes of being posted

8:38 AM

Tweet

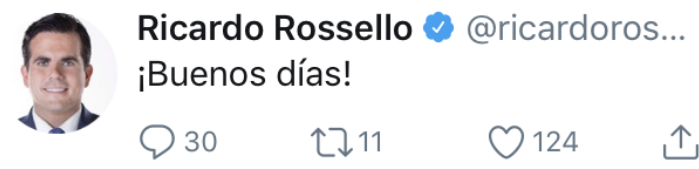

Panel B: Same tweet as Panel A one hour and 38 minutes after being posted

Figure 2: Good Morning! tweet by the former Governor of Puerto Rico posted at 7:30 AM and three minutes after it had 3 retweets and 17 likes (Panel A). After one hour and 38 minutes this tweet had 30 comments, 11 retweets, and 124 likes.

Given the role of social media during the protests that eventually led to the first resignation of an elected governor of Puerto Rico and how the Governor employed it, we sought to document on this work some of the interesting behavior we identified on Twitter around the principal hashtag for the protestors and counter-protestors. We also seek to study patterns in the followers of the former Governor's twitter account in light of the evidence brought forward by the forensic analysis discussed before.

\section{Data and Methods}

We collected data using the twitteR package and the data analysis was conducted in $\mathrm{R}$ (Gentry, 2016; RStudio Team, 2015). We monitored trends for Puerto Rico from July 12, 2019, and those containing information regarding the leak of the chat until the resignation of the Governor on July 25, 2019. Particularly, we tracked the hashtag \#RickyRenuncia ${ }^{1}$, which was the

\footnotetext{
${ }^{1}$ We recognize there are variants of the hashtag such as: \#RickyLeaks, \#RickyChats, \#RickyRenuncia, \#RickyVeteYa, \#RickyTeBote, \#RickyGameOver, \#RickyDictador, \#ArrestenAElíasSánchez, \#RickyRecoge, \#AbajoRosselló,
} 
first of many hashtags used to express discontent through social media. During our tracking, we identified the emergence of a countermovement with the hashtag \#RickySeQueda (which translates into Ricky Will Stay) which we also tracked until the July 25, 2019. In addition, we obtained data from the former Governor's verified twitter account (@ricardorossello) to study patterns in followers and online activity of these accounts that followed him. This data was collected using the rtweet package also in R (Kearney, 2019). The follower data is current as of August 13, 2019. The resulting dataset for this request provided information for approximately 150,000 accounts. We will discuss data cleaning and additional processes in the results section.

\section{Measurement}

\section{Clique and Community Detection}

Social media easily lends itself to be represented as a network of interactions. Throughout this paper, we will represent each Twitter user as a node, connecting to others by vertices, which can represent a "Retweet" or a "Mention" (Jackson \& Spencer, 2017). Network summary statistics were determined utilizing bibliometrix (Aria \& Cuccurullo, 2017). A 'clique' is defined as any given subgraph which is deemed 'complete', that is to say, every pair of distinct vertices in the subgraph is connected by a unique edge (see Figure 3).

Whereas cliques are explicitly defined, communities are a more general concept, with no agreed upon definitions. There is consensus, however, in that a community corresponds to a group of nodes that are similar enough to each other, and dissimilar from the rest of the network, as to merit their grouping together from the rest. For our study, we implemented an algorithm developed by Raghavan and colleagues (Raghavan, Albert, \& Kumara, 2007), where every node is initialized with a unique label, and iteratively, each node adopts the label most of its neighbors currently have, until consensus is reached.

\#ResidenciamientoYa, \#RickyRenunció. We limit our analysis to the first hashtag associated with this social movement, and the subsequent countermovement.

These are discussed by Torres-Lugo in the following news article: https://www.noticel.com/english/-rickyrenunciahow-social-media-helped-take-down-puerto-ricos-governor/1100593161 


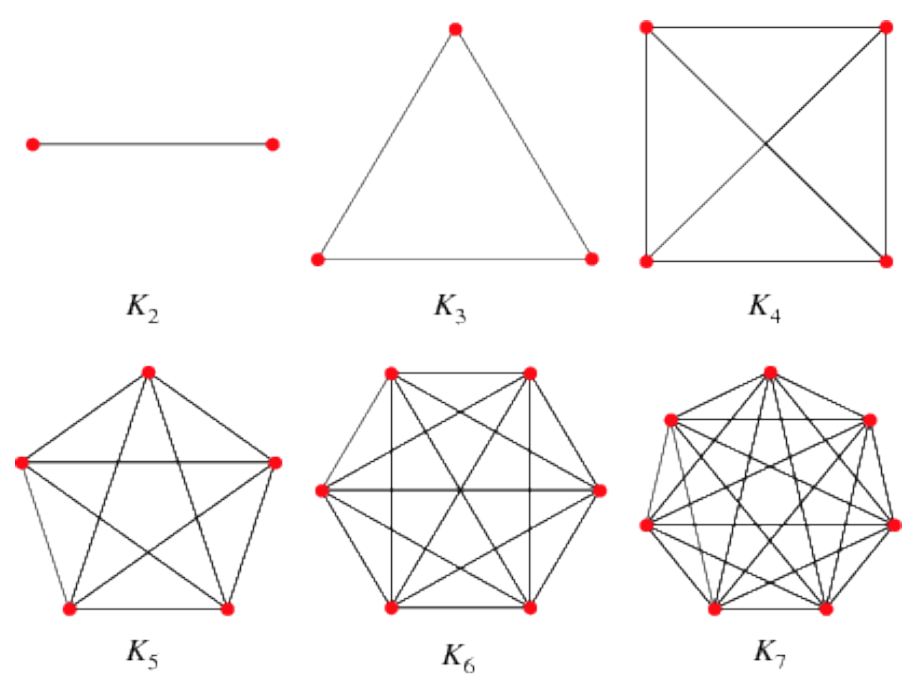

Figure 3: Examples of compete graphs, when presented as subgraphs known as cliques. A discussion of this topic if available in (G. Chartrand, 1985; R. L. Chartrand, 1985)

\section{Authorities and Hubs}

While Cliques and Communities are generally unobservant of directionality of information flow, it is useful to consider to what degree specific users influence the network structure and how. Seminal work on this area has defined the authority and hub scores based on the network's adjacency matrix (Kleinberg, 1999). For any given graph with adjacency matrix $A$, we may define the authority scores $a$ and hub scores $b$ as the eigenvectors of $t(A) * A$ and $A * t(A)$, respectively.

In the context of Twitter, for the standard definitions of Hubs and Authorities to apply. The vertex that connects user A with user B, where user B retweets user A, should, counterintuitively be considered an in-degree towards user A. In this manner, users with a higher authority score were those who generate significant activity in the form of retweets, whilst users with higher hubs scores were those that selectively retweeted users with significant authority scores. Additional information about the definitions and measurements discussed in this paper are available in previous literature and are beyond the scope of our line of inquiry (Heathrow \& Roman, 2016).

\section{Follower Analysis}

For each of the followers of the former Governor we produced five main indicators: 1) number of followers, 2) number of friends, 3) favorite count, 4) tweets count, and 5) age of the account. An overview of the anatomy of a tweet and specific indicators can be found in previous literature (Jackson \& Spencer, 2017), but we will discuss these indicators briefly in the Ricardo's Online followers section of this manuscript ${ }^{2}$.

\footnotetext{
${ }^{2}$ Additional information regarding the analysis of followers is available in tutorials such as the one produced by Benjamin Bellman, which is available here: https://rpubs.com/ben_bellman/rtweet_tidygraph
} 


\section{Results}

By the time, the Governor resigned approximately 1 million tweets calling for his resignation were generated under the hashtag \#RickyRenuncia (Panel A in Figure 4). In contrast, those calling for him to remain, under \#RickySeQueda, although reported by Twitter to have been trending at one point, barely reached 6000 tweets (Panel B in Figure 4). The obvious magnitude of differences validates what was reflected in public opinion, that the Governor was overwhelmingly being repudiated. Although these figures look similar, the axes were adjusted, in Panel A, the count is presented as hundred thousands, whereas in Panel B we present the raw counts..

A
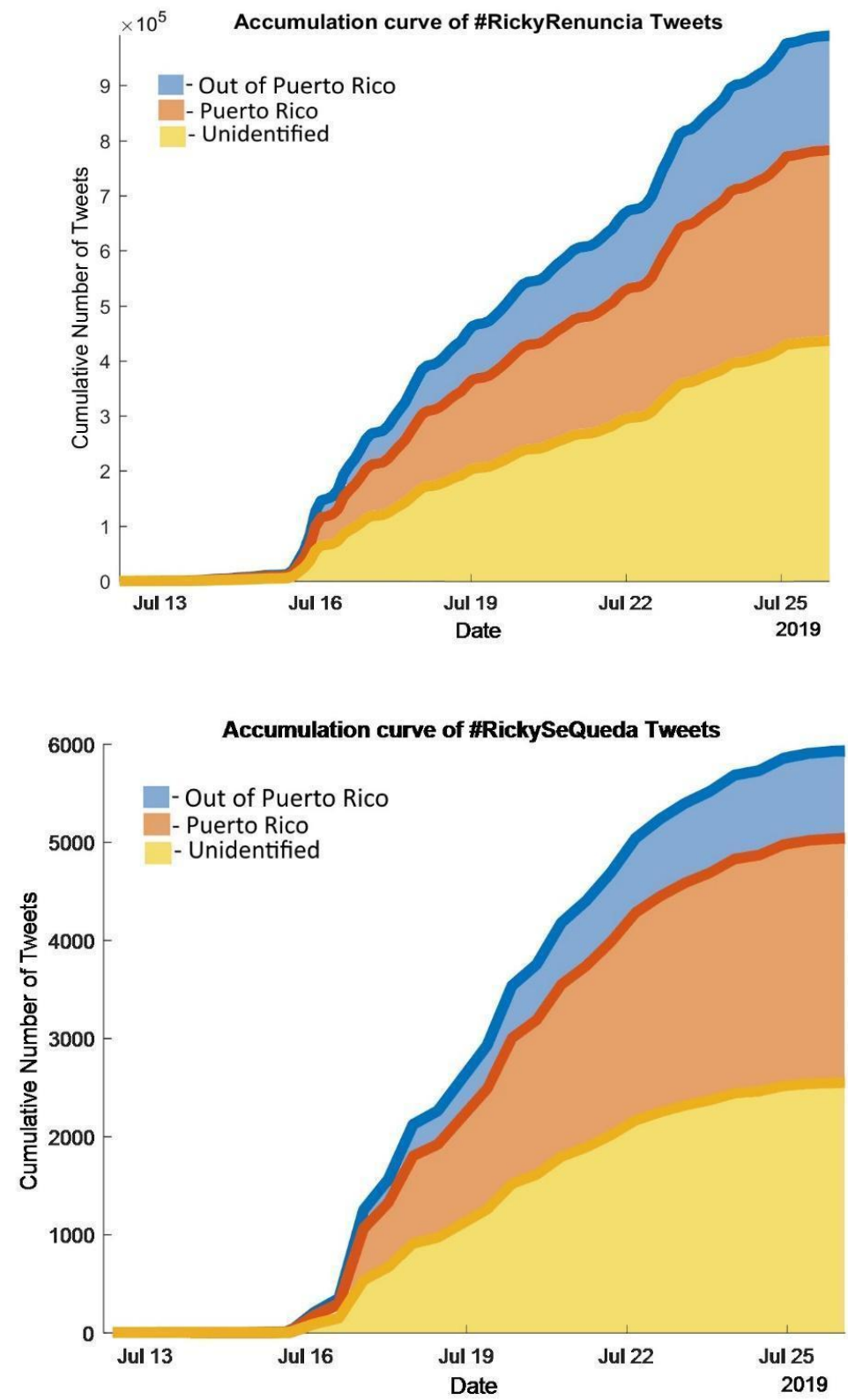

Figure 4: Accumulation curve of mentions of the hashtag \#RickyRenuncia (in thousands) and \#RickySeQueda (in raw counts) between Friday, July 12, 2019 and Wednesday, July 25, 2019. 


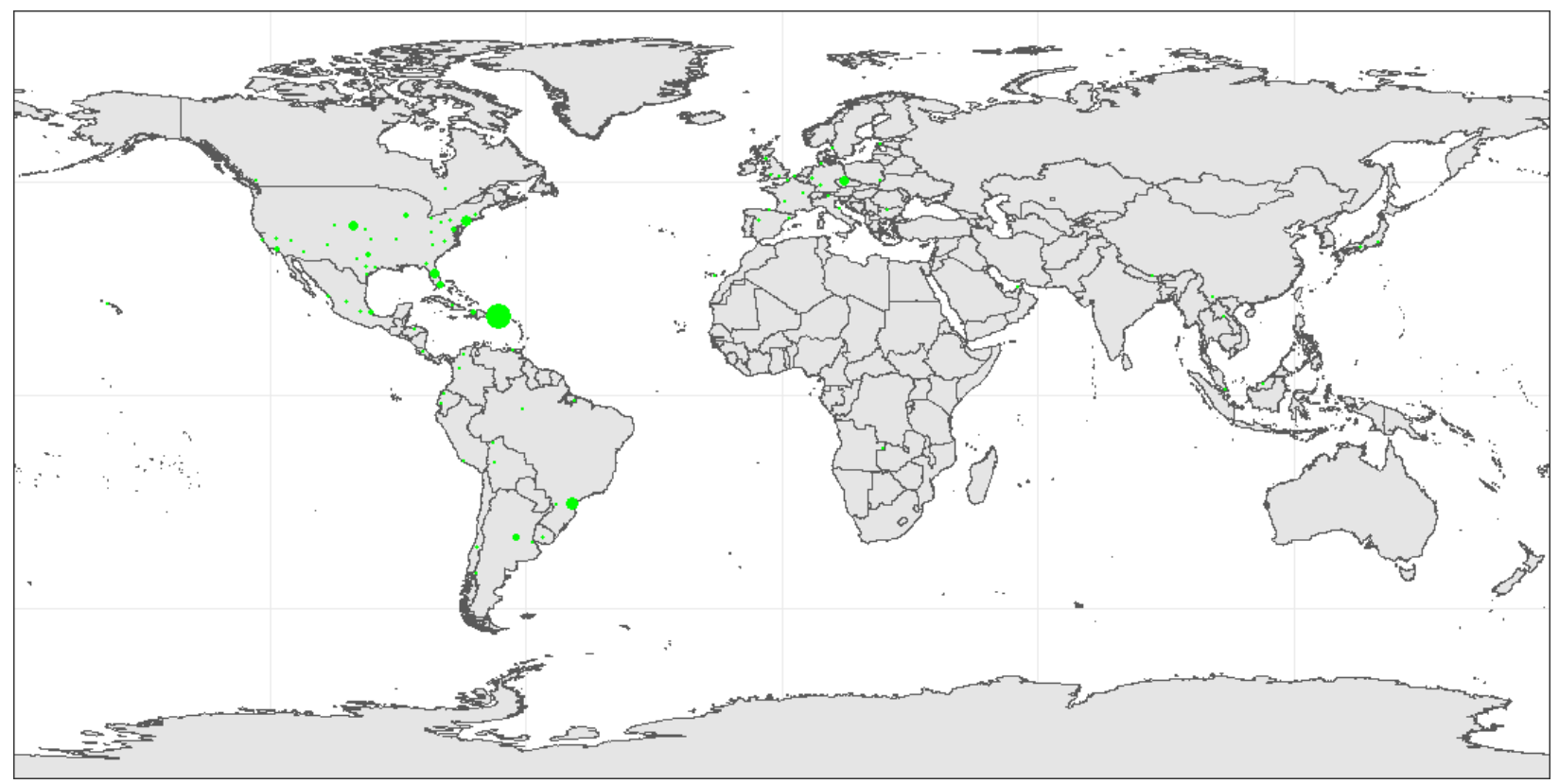

(a) \#RickyRenuncia

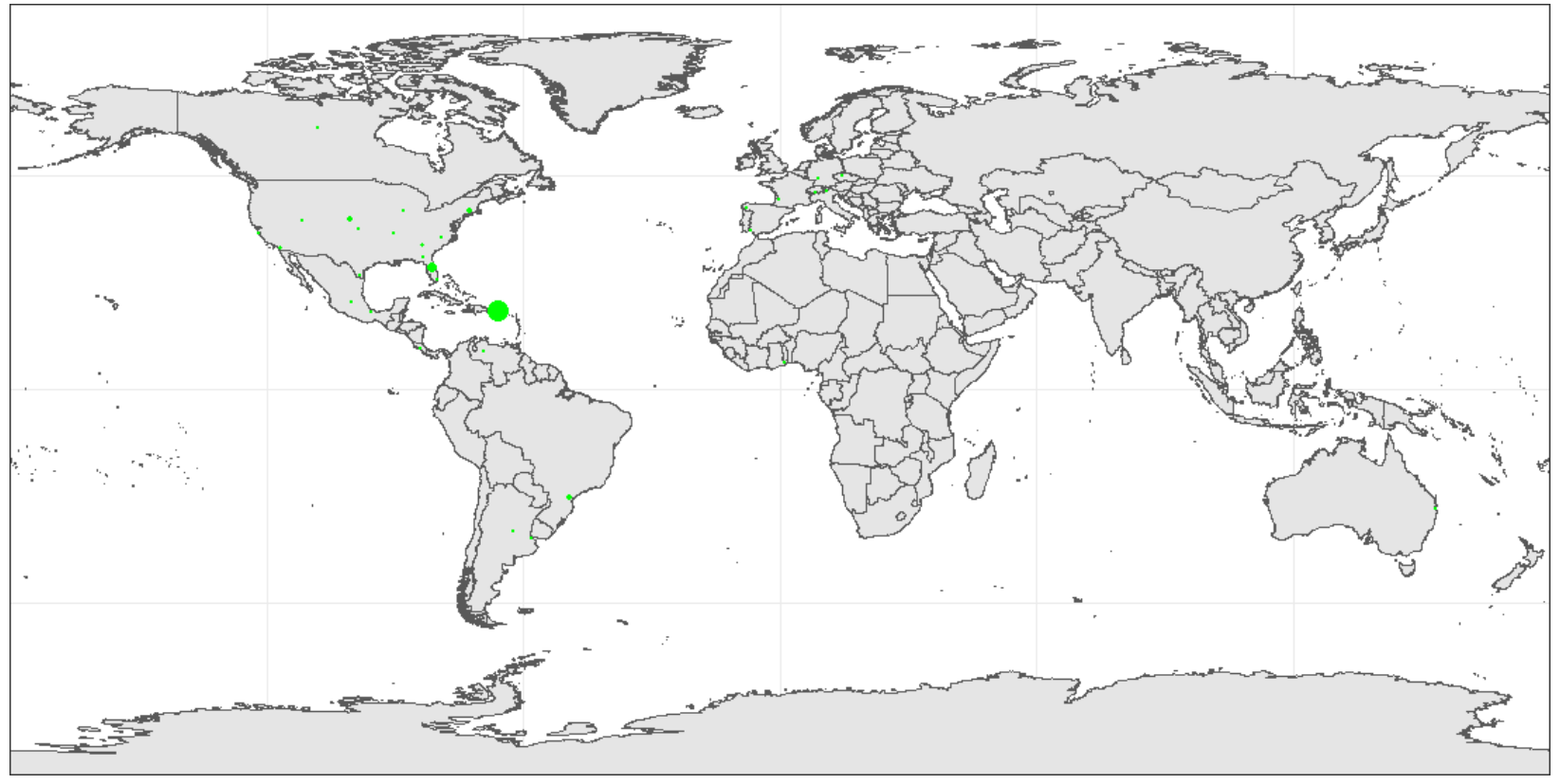

(b) \#RickySeQueda

Figure 5. Geographic distribution of \#RickyRenuncia (a) and \#RickySeQueda (b) hashtag usage in its initiation for the first 1,000 interactions.

In Figure 5, we present the geographic distribution of the first 1,000 interactions for each hashtag. We observe a significant dispersion of the hashtag use by geographical area, with the size of the dot representing the number of times it was used in that place. We must raise the caveat that in line with Figure 4, some tweets did not include any geocode or included geocodes that could not be translated into geographic locations (i.e. "Macondo", "en mi mundo", etc.). 
\#RickyRenuncia was used across the United States, but mostly in Puerto Rico and the USEast Coast places that have a significant concentration of Puerto Ricans living outside of the island. Significant activity is also observed in Western Europe. On the other hand, the use of the hashtag \#RickySeQueda seems to be concentrated in Puerto Rico and the southeastern states (mostly Florida) which is an emerging destination for Puerto Ricans regarding migration dynamics (Velazquez-Estrada, Marazzi-Santiago, Orenstein-Cardona, \& Romero-Pagán, 2018). Of particular attention is that some bot accounts that have been identified as pro-government (bot like activity) are located in Florida (Bandeira et al., 2019).

\section{Network structure}

In Table 1, we present descriptive statistics for the first 1,000 retweets for the hashtags \#RickyRenuncia and \#RickySeQueda; these allow us to understand the network structures associated with the birth of each hashtag.

\begin{tabular}{|l|c|c|}
\hline \multicolumn{2}{|c|}{ Table 1. Descriptive statistics for the first 1,000 retweets from each hashtag } \\
\hline & \#RickyRenuncia & \#RickySeQueda \\
\hline Edges (Retweets) & 1000 & 1000 \\
\hline Vertices (Users) & 806 & 314 \\
\hline Edge Density & 0.0014 & 0.0069 \\
\hline Reciprocity & 0.0020 & 0.0737 \\
\hline Global Transitivity & 0.0059 & 0.1599 \\
\hline Diameter & 6 & 8 \\
\hline Mean Node Degree & 2.4814 & 6.3694 \\
\hline Standard Deviation Node Degree & 7.8561 & 17.1861 \\
\hline Skewness Node Degree & 14.8747 & 6.3836 \\
\hline Degree Centrality & 0.0863 & 0.1405 \\
\hline Average Shortest Path Length (APL) & 4.3280 & 3.6561 \\
\hline Mean Clique Size & 1.5644 & 3.0859 \\
\hline Note: Descriptive statistics derived from the data used to construct Figure 5 and Figure 6. \\
\hline
\end{tabular}

Differences are observed in the structure of both networks. First, the number of users differed by hashtag the first 1,000 mentions of \#RickyRenuncia involved 806 users, whereas the first 1,000 uses of \#RickySeQueda involved 314 users. This means that at the beginning of each movement more users were involved in \#RickyRenuncia than in the \#RickySeQueda movements. This is manifest in the significant differences in edge density, as \#RickyRenuncia is a demonstrably sparse network, in contrast with the very dense network of \#RickySeQueda. As discussed in the previous literature, the sparsity of the \#RickyRenuncia network is reflective of truly expansive usage, whilst the dense and closed patterns of \#RickySeQueda are unlikely to lead to proliferative usage (Huberman, Romero, \& Wu, 2009).

The network descriptive measures indicate that the users who engaged in \#RickyRenuncia were less connected than those who participated in the \#RickySeQueda movement (Mean Node Degrees $=2.48$ and 3.67, respectively). Further, both networks possess a level of skewness; however, the asymmetry is higher for the \#RickySeQueda hashtag. The \#RickyRenuncia 
engagement had a lower indicator of degree centrality (0.0863) than the \#RickySeQueda movement (0.1405), this is indicative that the \#RickySeQueda movement had higher level of influential persons who initiated the message and this message was spread by the other users. Contrary to this, the degree centrality for \#RickyRenuncia is indicative of less influence by individual users, and engagement of numerous persons. This is consistent with other indicators included in Table 1, and the visualization of these networks included in Figures 6 and 7.

Furthermore, as we compare the networks in terms of their transitivity and reciprocity, it becomes patently obvious that the usage of \#RickySeQueda corresponds to that of an echo chamber, such as described in empirical examinations of previous echo chambers (Jasny et al., 2018; Jasny, Waggle, \& Fisher, 2015). These measures capture the degree to which the network forms clusters, and users mutually reciprocate in their interactions, respectively. This is further validated by the correlation of Hub and Authority Scores with nodes that participate in increasingly large cliques (Figure 6). This tendency is not found by \#RickyRenuncia, as the formation of cliques, even as small as $\mathrm{K}=3$, was very infrequent.

This is effectively visualized in Figure 7, where the emergence of a clear community structure is present for \#RickySeQueda, whilst the community structure of \#RickyRenuncia is far from monolithic. While the Cluster Label Propagation algorithm effectively groups more than half of the nodes in the \#RickySeQueda network, less than $20 \%$ of the nodes for \#RickyRenuncia pertain to the largest community structure.
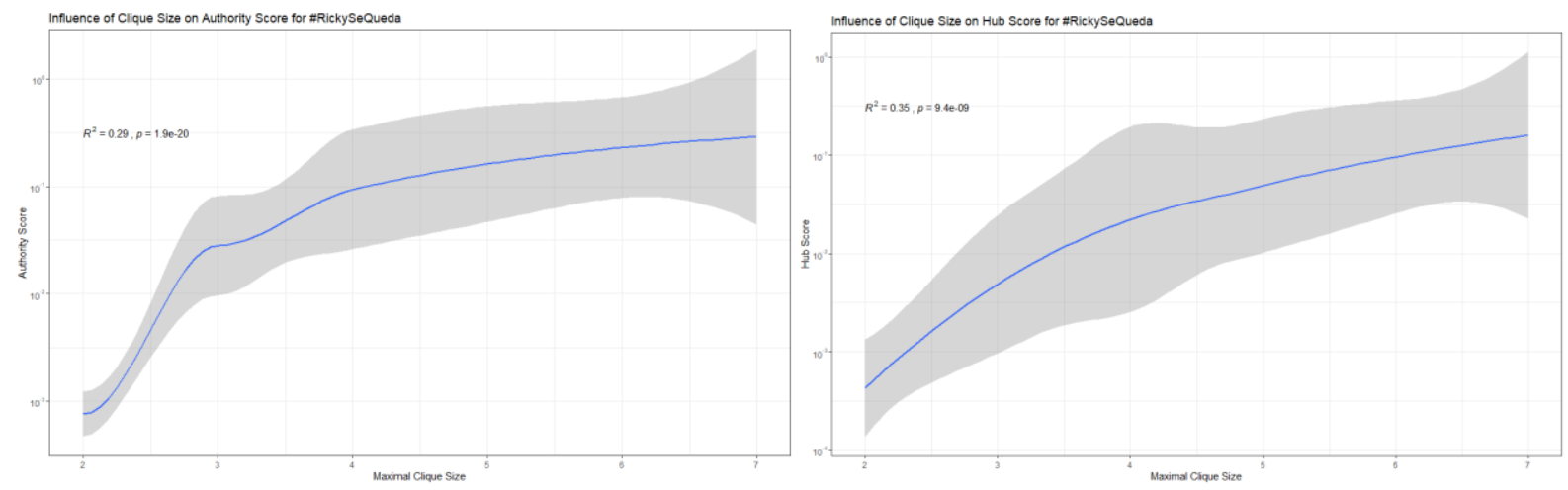

Figure 6: Correlation of Authority and Hubs scores utilizing the HITS algorithm, with the largest clique size in which a given node participated. 

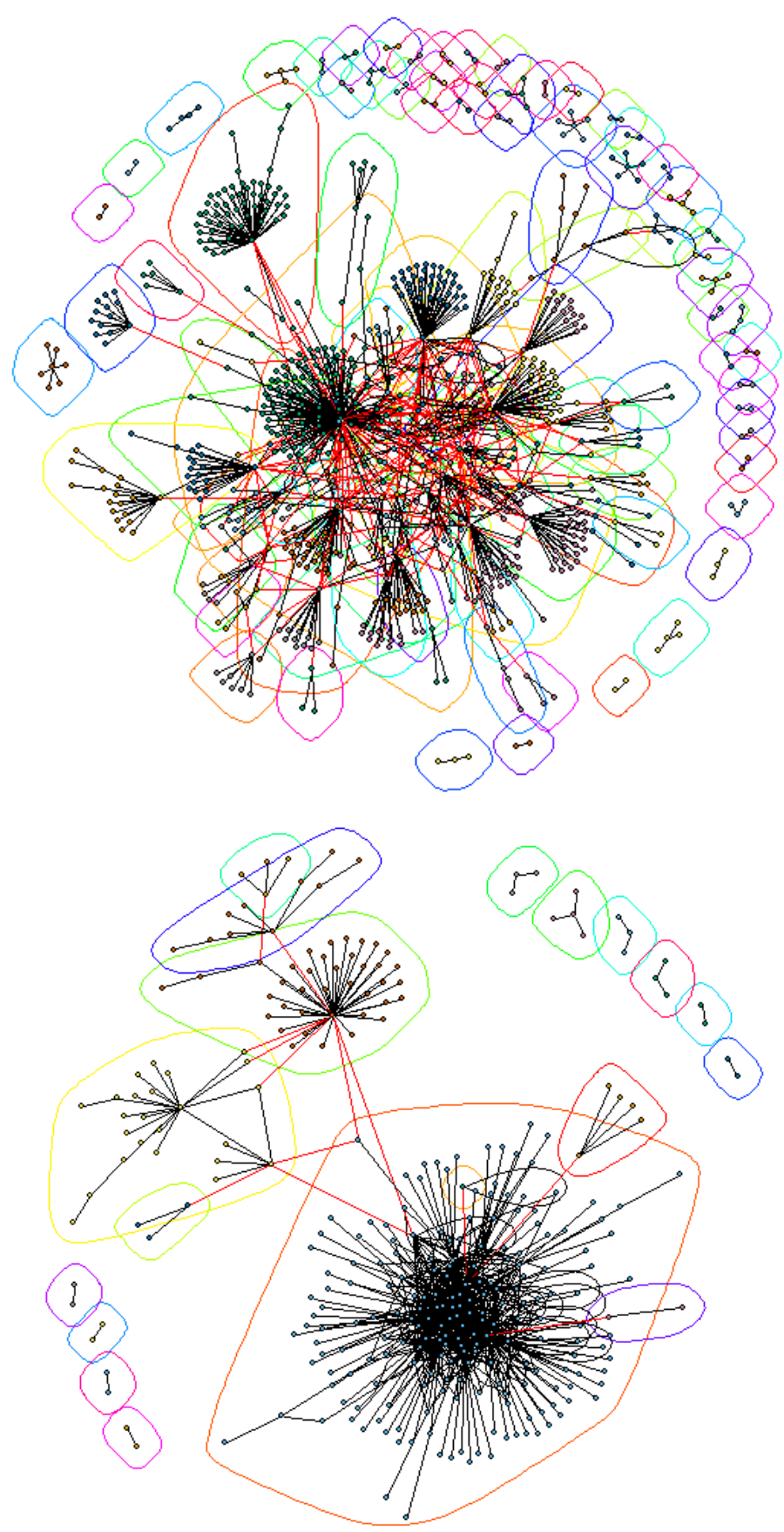

Figure 7: Community Structures of \#RickyRenuncia and \#RickySeQueda usage in its initiation using community label propagation. 


\section{Network Diffusion}

The hashtag \#RickySeQueda started circulating among governor-friendly accounts on the night of July 16, which was perceived by some protestors on Twitter as an attempt to create a trending topic to counter the \#RickyRenuncia hashtag. At around 1:00 AM on July 17, we used Hoaxy to extract the recent diffusion network for the \#RickySeQueda hashtag (Shao, Ciampaglia, Flammini, \& Menczer, 2016). We removed entries that were made by protestors mocking the attempt; which restricted the analysis with the set of governor-friendly accounts that were truly interacting with the hashtag.

A visualization of the diffusion network for both hashtags is shown in Figure 8 . The contrast between both networks is evident in the interaction between nodes. Whereas there is a small set of highly connected nodes in the \#RickySeQueda network, the edge connections in the \#RickyRenuncia network, outside of stars created by popular tweets being retweeted, are between a small numbers of disconnected nodes. This behavior seems suspicious in light of the discussions that were had between the Telegram chat participants that we previously mentioned. To determine whether our suspicion was unfunded or not, we examined the actions of the individual network actors.

The interactions in the \#RickySeQueda network were dominated by user mentions and retweets, 219 and 300, respectively. The retweets were made by 104 accounts, while the mentions were made by 30 accounts. We note that we removed mention rows that were duplicated to avoid inflating the number of accounts that were directly exposed to the hashtag. Figure 9 contains boxplot summarizing the number of times these accounts interacted with the hashtag.

The top mention outlier was an account (@mlamboy15) that mentioned 46 other accounts and was identified by the DFRLab as having frequently participated in the campaigns coordinated through the Telegram chat. On the other hand, three of the retweets outliers (@maramora, @1uboso1966,@BebaDril7) were included in the DFR's analysis as having a high eigenvector centrality (Bandeira et al., 2019). On Figure 10, we present a cumulative plot of all the tweets that were sent from these accounts and highlight the fact that there is an inflection point at around 8:00 PM in which the number of tweets increased exponentially.

While we cannot make a definitive statement whether any of the accounts that shared the \#RickySeQueda hashtag are paid trolls, we do note that the behavior displayed by these accounts seems irregular and that most of these accounts continue publishing government- or party-friendly tweets. It at least is indicative of a coordinated effort by these accounts or a coincidence that these users decided to tweet about this topic at the same time; our conclusion is in line with that of Bandeira and colleagues (2019). This finding is consistent with the two previous analyses on this matter (Bandeira et al., 2019; Vicens, 2019). 


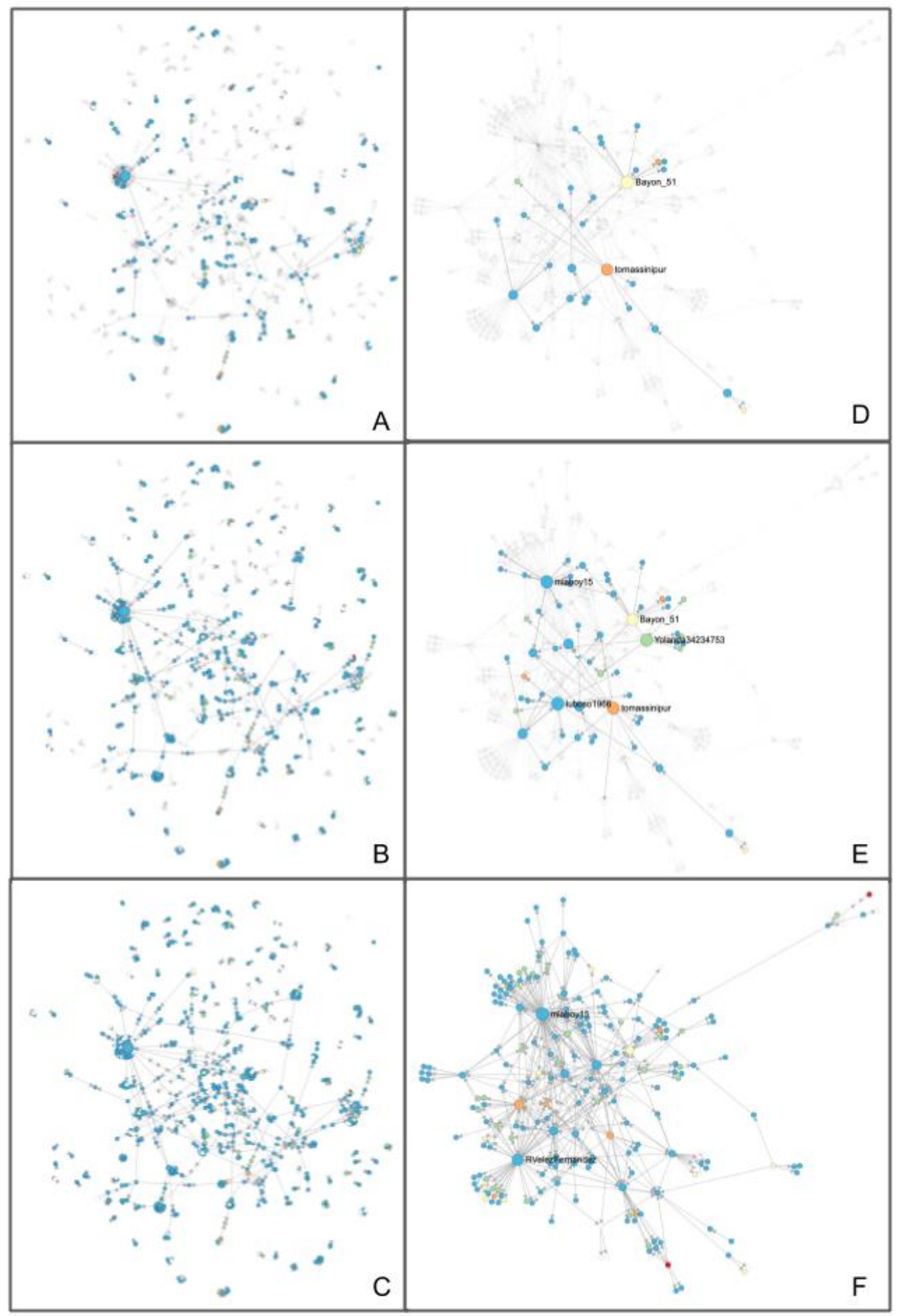

Figure 8: Diffusion network for \#RickyRenuncia (Panels A to C) and \#RickySeQueda (Panels D to F) between 10:12 AM and 10:21 AM in July 17, 2019 

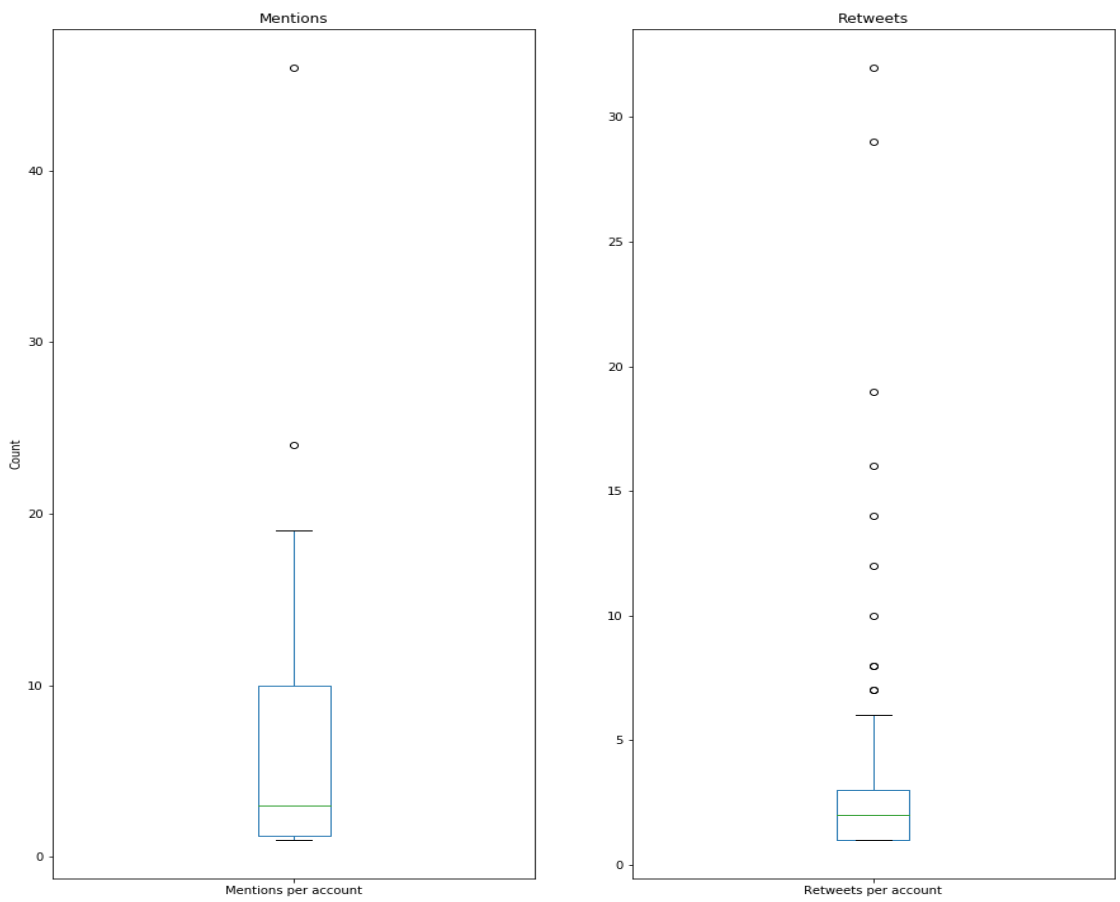

Figure 9. Boxplot summary of the total number of interactions per account for the hashtag \#RickySeQueda
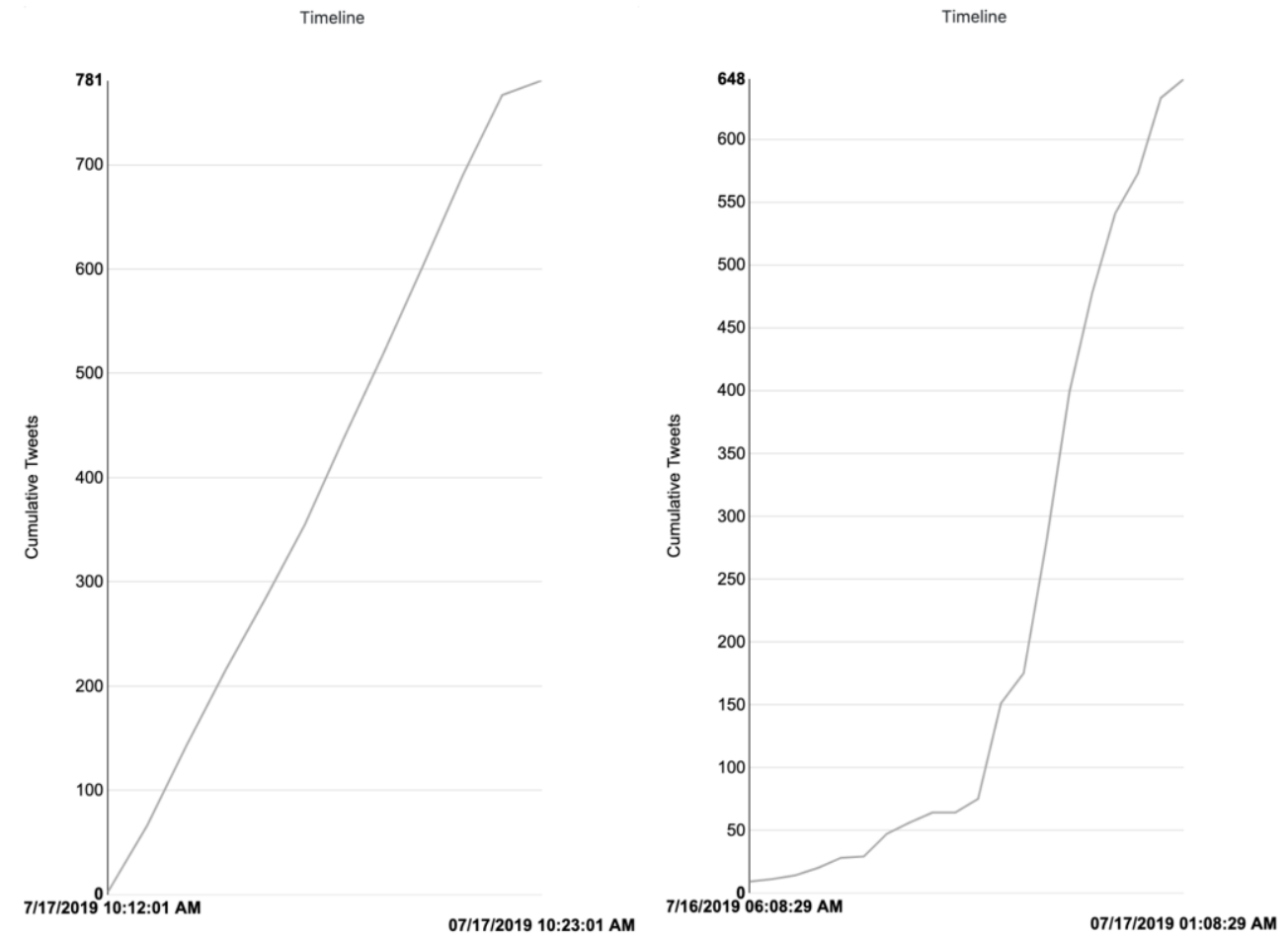

Figure 10. Cumulative tweet plot for \#RickyRenuncia (left) between 10:12 AM and 10:21 AM in July 17, 2019 and \#RickySeQueda (right) between 06:08 PM and 01:08 AM in July 16-17, 2019 


\section{Patterns of interactions}

In Figures 11 and 12, we present networks structures for \#RickyRenuncia and \#RickySeQueda between July 12 to July 17. In Figure 11, we present the \#RickyRenuncia network and found communication between more than 15,000 users and encompassing 33,720 edges (filtering with a $\mathrm{K}$ score of 7). Of particular attention is the red box in Figure 11, in which we find official government accounts and accounts for government officers who were mentioned in the tweets but did not engage in the use of the \#RickyRenuncia hashtag during the period of analysis. The most mentioned account was that of the Governor of Puerto Rico (@ricardorossello) followed by the official account of the Government (@fortalezapr). These two accounts are closer to the overall network, located at the bottom-left area of the red box.

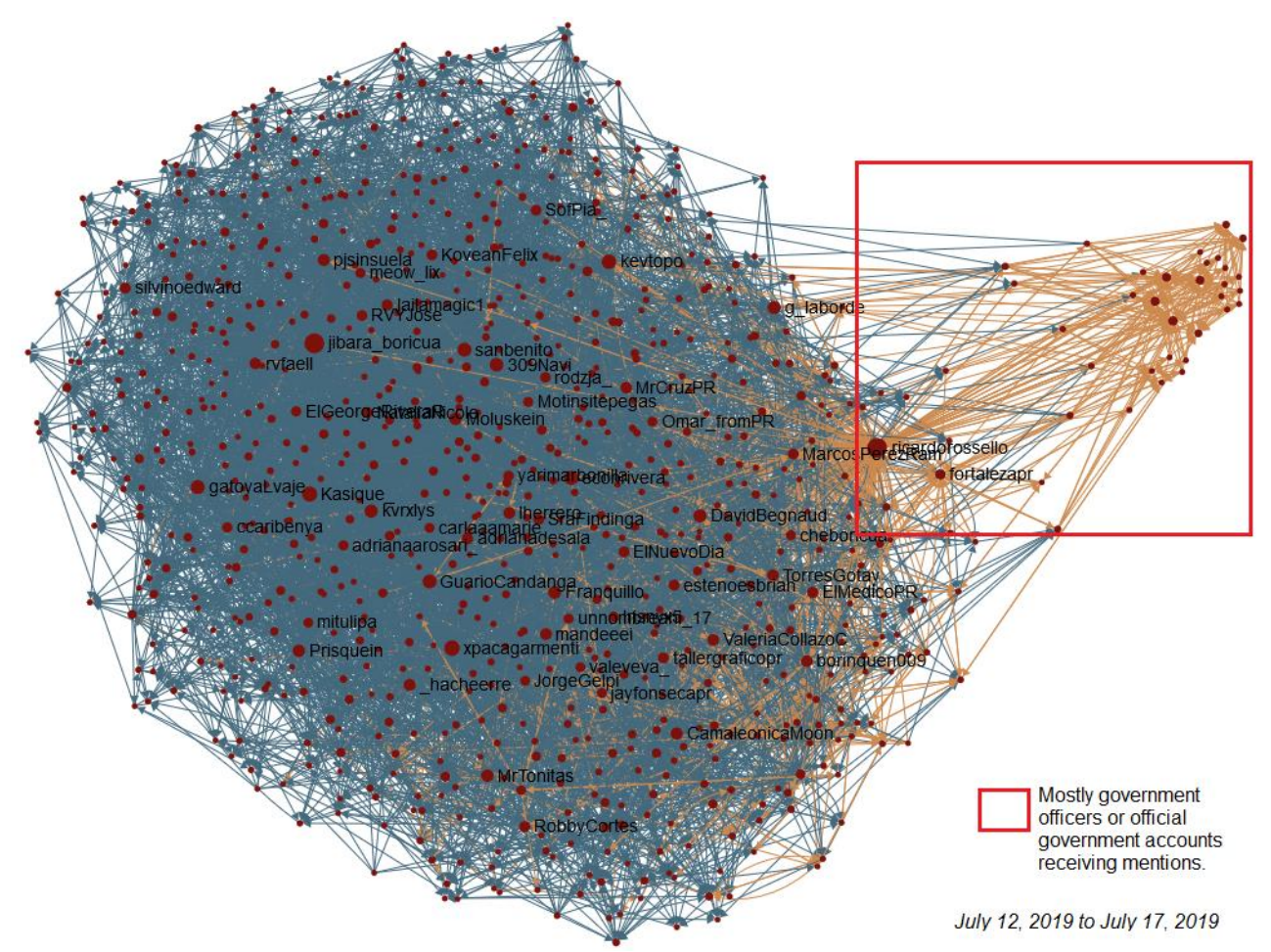

Figure 11: Interactions for \#RickyRenuncia between July 12, 2019 and July 17, 2019.

In Figure 12, we show the structure for \#RickySeQueda for the same period. In this network, we found engagement of 253 users, encompassing 407 edges (no filter was applied). A significant part of these accounts have been identified as "bots", "trolls" or "fotutos" in forensic analysis conducted by the Digital Forensic Research Lab (Bandeira et al., 2019) and other independent analyses(Vicens, 2019). This is indicative of low level of support among the population for the \#RickySeQueda movement, mostly concentrated in accounts that have been labeled as propagators of the message and agenda of the government agenda set forth by Dr. Ricardo Rosselló and his public relations team. 


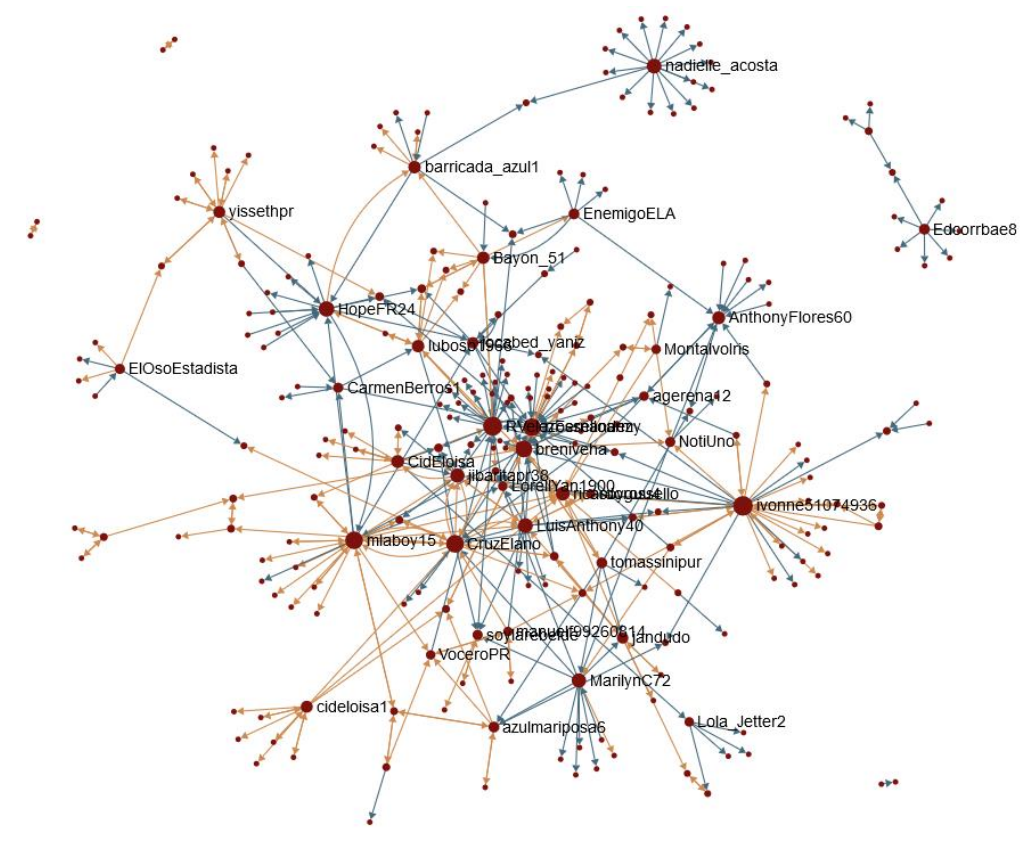

Figure 11: Interactions for \#RickySeQueda between July 12, 2019 and July 17, 2019.

\section{Ricardo's online followers}

As mentioned before, using rtweet we accessed information about the accounts who followed Dr. Ricardo Rossello on his official social media account (Kearney, 2019). The initial data request provided 150,000 accounts with up to 90 variables associated with each account. A close inspection of the number of followers indicated that the distribution of followers of these accounts was being skewed by approximately 20 accounts. We excluded every account, which had been verified by Twitter from the analytical pool, as these were more likely to be: companies, celebrities, government officers, news networks, and other politicians. This reduced our analytical sample to 148,928 users. First, we were interested in establishing a profile of those who composed the digital network of the former Governor. In Table 2, we present the descriptive statistics for the followers of the former Governor.

\begin{tabular}{|c|c|c|c|c|}
\hline & Mean & Median & $1^{\text {st }}$ Quartile & $3^{\text {rd }}$ Quartile \\
\hline Followers & 315 & 19 & 4 & 94 \\
\hline Friends & 594 & 177 & 65 & 531 \\
\hline Favorite Count & 2844 & 44 & 3 & 589 \\
\hline Tweets Count & 3194 & 47 & 4 & 644 \\
\hline Age of Account (in years) & 5 & 4.9 & 2 & 7.8 \\
\hline
\end{tabular}

We found that $25 \%$ of the accounts that followed Ricardo Rossello had less than 4 followers, and $50 \%$ of them had less than 20 followers. We also found that $25 \%$ of these counts had less than 65 friends (persons who follow each other mutually) and about $50 \%$ of them had less than 177 friends. In addition, about $25 \%$ of the accounts had 3 favorite tweets or less, and $50 \%$ of 
these followers had less than 44 favorite tweets. Further, and consistent with previous indicators, there is a distinct trend also in the number of tweets by account where $25 \%$ of the accounts that follow the former Governor had less than 4 tweets, and about $50 \%$ of these accounts had less than 48 tweets. Finally, in this overall analysis we found that the accounts have an average digital age in years of 5 , with a median of 4.9 years. $25 \%$ of the accounts have a digital age of less than two years, while $75 \%$ of the accounts have a digital age lower than 7.8 years (created after 2011).

Upon closer examination to this descriptive statistics, we found that the accounts that follow the person of interest could be divided in two groups: 1) those who are active and 2) those whose accounts remain dormant. We classified the accounts based on the following criteria: the accounts were active if they tweeted at least once every two months, and they were considered active if they tweeted more frequently than once every two months. We produced descriptive statistics for these accounts, which are presented in Table 3 . The descriptive analysis by categories indicates that indeed, two groups that have different characteristics follow the Governor. In all indicators presented in Table 2, those who are active have indicators that point towards accounts that behave organically, whereas the dormant accounts have lower numbers of followers, friends, favorites, tweets, and digital ages. Underlying patterns within these populations should be studied in future research.

\begin{tabular}{|c|c|c|c|c|}
\hline & Mean & Median & $1^{\text {st }}$ Quartile & $3^{\text {rd }}$ Quartile \\
\hline \multicolumn{5}{|c|}{ Dormant Accounts (less than 1 tweet every 2 months, $n=61,292)$} \\
\hline Followers & 15 & 4 & 1 & 13 \\
\hline Friends & 177 & 74 & 32 & 158 \\
\hline Favorite Count & 113 & 0 & 0 & 16 \\
\hline Tweets Count & 7 & 2 & 0 & 9 \\
\hline Age of Account (in years) & 4.3 & 3.6 & 1.9 & 6.5 \\
\hline \multicolumn{5}{|c|}{ Active Accounts (more than 1 tweet every 2 months, $n=87544$ ) } \\
\hline Followers & 525 & 61 & 16 & 232 \\
\hline Friends & 885 & 344 & 141 & 908 \\
\hline Favorite Count & 4759 & 309 & 45 & 2020 \\
\hline Tweets Count & 5425 & 387 & 84 & 2360 \\
\hline Age of Account (in years) & 5.6 & 5.8 & 2.5 & 8.5 \\
\hline
\end{tabular}

\section{Discussion}

According to the Pew Research Center, $62 \%$ of U.S. adults get their news on social media (Gottfried \& Shearer, 2016). Recent research indicates that registered voters are exposed and are bound to share fake information due to their political leanings, or because they do not understand the existence of misinformation on social media (Friedland, Joseph, Swire-Thompson, Grinberg, \& Lazer, 2019). As the years have gone by, more and more has it been shown that the impact social media has on our politics grows as days pass by. Greatly expanding the common citizen's access 
to information, as well as offering platforms to actors who may not traditionally harness much political power, social media is rapidly transforming our democratic processes (Highfield, 2017). The citizenry expects greater transparency, authenticity and real activity. Leaders unable to rise to these standards are bound to lose the trust of their people they serve.

Our exploration of the case of El Verano del 19 was focused on identifying the key characteristics of the antagonistic political movements, \#RickyRenuncia and \#RickySeQueda. It is clear that the decentralization of the \#RickyRenuncia movement was the determinant characteristic that led to its significantly greater proliferation than \#RickySeQueda. Moreover, we underlined the futility of the actions of "key" actors that only strengthened the nature of the \#RickySeQueda movement as an isolated echo chamber.

In light of those "key" actors we want to note that while much emphasis has been given in the political discourse and literature to interference in election and governmental affairs from nationstate or state-sponsored actors on social media, this work documents what seems to be a continuation of a public opinion manipulation campaign by suspicious accounts that might be acting in coordination with government officials. Unlike previous attempts, these accounts seem to be troll-like and exhibit a low botscore from bot detection tools such as Botometer, which makes them hard to isolate. Nonetheless, the accounts do seem to exhibit irregular behavior as we shown in this article. By documenting this case, we hope to provide clues that can help in the development of tools that can identify this type of behavior in the future.

\section{Acknowledgments:}

To the University of Puerto Rico for educating the three of us, something that is becoming more difficult with the approval of austerity measures in Puerto Rico. ARSL is a Faculty Affiliate of the Social Science Research Institute (SSRI) at the Pennsylvania State University. We are grateful to the University of Indiana Bloomington and the Observatory on Social Media (https://osome.iuni.iu.edu/) for providing us with the tools used to produce part of our analysis. To the people of Puerto Rico for defending the country and exercising their right to protest to shape the future of the island.

\section{References:}

Anduiza, E., Cristancho, C., \& Sabucedo, J. M. (2014). Mobilization through online social networks: the political protest of the indignados in Spain. Information Communication and Society. https://doi.org/10.1080/1369118X.2013.808360

Aria, M., \& Cuccurullo, C. (2017). bibliometrix: An R-tool for comprehensive science mapping analysis. Journal of Informetrics. https://doi.org/10.1016/j.joi.2017.08.007

Bandeira, L., Ponce de León, E., \& Clark, S. (2019). From Telegram to Twitter: Top Puerto Rican Officials Plotted Possible Information Operation. Washington, D.C. Retrieved from https://medium.com/dfrlab/from-telegram-to-twitter-top-puerto-rican-officials-plottedpossible-information-operation-a899a00e078e

Chartrand, G. (1985). Introduction Graph Theory. (Dover, Ed.). 
Chartrand, R. L. (1985). The politics of information. Journal of the American Society for Information Science. https://doi.org/10.1002/asi.4630360606

Friedland, L., Joseph, K., Swire-Thompson, B., Grinberg, N., \& Lazer, D. (2019). Fake news on Twitter during the 2016 U.S. presidential election. Science, 363(6425), 374-378. https://doi.org/10.1126/science.aau2706

Gentry, J. (2016). Package “twitteR”: R Based Twitter Client. CRAN.

Gottfried, J., \& Shearer, E. (2016). News use scross docial media platforms 2017. Pew Research Center. Retrieved from https://www.journalism.org/2016/05/26/news-use-across-socialmedia-platforms-2016/

Heathrow, L., \& Roman, T. (2016). Node Degree and Strength. Fundamentals of Brain Network Analysis, 115-136. https://doi.org/10.1016/b978-0-12-407908-3.00004-2

Highfield, T. (2017). Social media and everyday politics. John Wiley \& Sons.

Huberman, B. A., Romero, D. M., \& Wu, F. (2009). Social networks that matter Twitter under the microscope. First Monday.

Jackson, M. D., \& Spencer, S. (2017). Engaging for a Good Cause: Sophia's Story and Why \#BugsR4Girls. Annals of the Entomological Society of America. https://doi.org/10.1093/aesa/sax055

Jasny, L., Dewey, A. M., Robertson, A. G., Yagatich, W., Dubin, A. H., Waggle, J. M. C., \& Fisher, D. R. (2018). Shifting echo chambers in US climate policy networks. PLoS ONE. https://doi.org/10.1371/journal.pone.0203463

Jasny, L., Waggle, J., \& Fisher, D. R. (2015). An empirical examination of echo chambers in US climate policy networks. Nature Climate Change. https://doi.org/10.1038/nclimate2666

Kearney, M. W. (2019). Package "rtweet": Collecting Twitter Data. CRAN. Retrieved from https://cran.r-project.org/web/packages/rtweet/rtweet.pdf

Kleinberg, J. M. (1999). Authoritative sources in a hyperlinked environment. Journal of the ACM. https://doi.org/10.1145/324133.324140

Raghavan, U. N., Albert, R., \& Kumara, S. (2007). Near linear time algorithm to detect community structures in large-scale networks. Physical Review E - Statistical, Nonlinear, and Soft Matter Physics. https://doi.org/10.1103/PhysRevE.76.036106

RStudio Team. (2015). RStudio: Integrated Development for R. RStudio.

Shao, C., Ciampaglia, G. L., Flammini, A., \& Menczer, F. (2016). Hoaxy: A platform for tracking online misinformation. In Proceedings of the 25th International Conference Companion on World Wide Web. International World Wide Web Conferences Steering Committee.

Valentín Ortiz, L. J., \& Minet, C. (2019). Las 889 páginas de Telegram entre Rosselló Nevares y sus allegados. Retrieved September 8, 2019, from http://periodismoinvestigativo.com/2019/07/las-889-paginas-de-telegram-entre-rossellonevares-y-sus-allegados/ 
Varnali, K., \& Gorgulu, V. (2015). A social influence perspective on expressive political participation in Twitter: the case of \#OccupyGezi. Information Communication and Society, 18(1), 284-304. https://doi.org/10.1080/1369118X.2014.923480

Velazquez-Estrada, A. L., Marazzi-Santiago, M., Orenstein-Cardona, J., \& Romero-Pagán, N. M. (2018). Perfil del Migrante. Hato Rey, Puerto Rico: Puerto Rico Institute of Statistics.

Vicens, A. (2019, July 29). How Puerto Rico's Governor Used a Secret Chat Group to Guide a Bot Network Attacking His Opponents. MotherJones.

Wolfsfeld, G., Segev, E., \& Sheafer, T. (2013). Social Media and the Arab Spring: Politics Comes First. International Journal of Press/Politics. https://doi.org/10.1177/1940161212471716 